DOI: $10.21767 / 2572-5483.100010$

\title{
Towards Evidence-Informed Policymaking in Physical Activity
}

\section{Arja R Aro}

Unit for Health Promotion Research, University of Southern Denmark, Esbjerg, Denmark

Corresponding author: Arja R Aro, Unit for Health Promotion Research, Professor of Public Health, University of Southern Denmark, Esbjerg, Denmark, Tel: +45 60111874; E-mail: araro@health.sdu.dk

Rec date: August 11, 2016; Acc date: August 12, 2016; Pub date: August 18, 2016

Copyright: (c) 2016 Aro AR. This is an open-access article distributed under the terms of the Creative Commons Attribution License, which permits unrestricted use, distribution, and reproduction in any medium, provided the original author and source are credited.

Citation: Aro AR (2016) Towards Evidence-Informed Policymaking in Physical Activity. J Prev Med 1: 18.

Keywords: Evidence-informed; Policymaking; Physical Activity; Public Health; Health promotion

There is strong evidence for physical activity enhancing health; also prominent calls were presented in the recent Lancet Series for Physical Activity to build policies and supporting daily living environment to sustain physical activity [1]. Papers also in this journal deal with interventions to increase physical activity $[2,3]$.

Even when we do have accumulating research evidence on contextual factors being important to physical activity, it has proved difficult to integrate research evidence with real-life policymaking. One reason for this is that compared to clinical field mostly dealing with individuals, in policymaking, research evidence is seldom the driving force; instead, 'other kinds of evidence' such as local population characteristics, local values, priorities, and resources play an important role [4]. Applying research evidence to enhance physical activity in policies e.g. building a bicycle lane in a city needs to happen in collaboration and on equal footing between research producers and policy stakeholders. This kind of collaborative policymaking is called evidence-informed instead of evidence-based, meaning that research evidence only informs policymaking.

\section{Research to enhance evidence-informed policymaking}

There is very little research evidence to show how best to develop policies in the evidence-informed way. A seven country (Denmark, Italy, Finland, the Netherlands, Romania, UK; Canada as evaluator) project Research into Policy to enhance physical activity (REPOPA; www.repopa.eu (European Commission grant No 281532; 2011-2016) took this challenge to study how best to integrate research evidence into real-life policymaking in the evidence-informed way [5].

In the REPOPA project, use of research and other kinds of evidence in 21 physical activity policies in six European countries was analyzed and 86 policy stakeholders were interviewed to find facilitators and barriers for research evidence use in policymaking [5-7].
Building on the findings, two sets of interventions, both implemented in three countries, were developed. One set used policy gaming with 18-19 researchers and policymakers in each of the three games to increase inter-sectoral collaboration in locally salient physical activity policymaking scenarios; these interventions increased understanding of how to strengthen the evidence-informed policymaking especially in cross-sector collaboration [8]. The other set of interventions aimed at increasing evidence-informed policymaking in six stewardship and needs-based locally tailored interventions ( $n=74$ stakeholders) on physical activity policymaking [9]. These interventions increased taking into account research knowledge, priorities and values of policy target groups in policy development. The preliminary intervention results have been reported to the REPOPA funder (see www.repopa.eu for Deliverables D2.2., D 3.2.; manuscripts are in preparation).

Further, based on the project results, an international tworound Delphi ( $n=76$ and 72 participants respectively) and six national conferences in the European REPOPA partner countries were used to agree, validate and contextualize measurable evidence-informed policymaking indicators (www.repopa.eu). These indicators and policy briefs being developed in the project will provide practical tools both for researchers and policymakers to work evidence-informed.

\section{Challenges in studying real-life policymaking}

Studying real-life policies in different contexts is a challenge due to natural and uncontrollable changes in policies and among policymakers. Another challenge is that this kind of research on developing salient and applicable policy interventions for communities has to be needs-based and tailored to the local reality. To systematically learn from contextual policy research, there is an urgent need to increase resources for research done in these natural settings. In addition, scientific journals could be more open to publish these implementation research reports even if they do not adhere to the prevailing paradigm of strict control of research conditions. 


\section{References}

1. Reis RS, Salvo D, Ogilvie D, Lambert EV, Goenka S, et al. (2016) Scaling up physical activity interventions worldwide: stepping up to larger and smarter approaches to get people moving. The Lancet.

2. Yu CY, Zhu X (2016) From attitude to action: What shapes attitude toward walking to/from school and how does it influence actual behaviors? Prev Med 90: 72-78.

3. Gondim PFJG, Alvin MSM, da Conceicaao AM, de Carmen BMM, Aquino EML, et al. (2016) Factors associated with leisure time physical activity among ELSA-Brasil participants: Ecological model. Prev Med 90: 17-25.

4. Aro AR, Smith J, Dekker J (2008) Contextual evidence in clinical medicine and health promotion. Eur J Public Health 18: 548-549.

5. Aro AR, Bertram M, Hämäläinen R-M, Van de Goor L, Skovgaard T, et al. (2015) Integrating research evidence and physical activity policy making - REPOPA project. Health Promotion International 31: 1-10.

6. Hämäläinen R-M, Aro AR, van de Goor L, Juel Lau C, Jakobsen $M W$, et al. (2015) Exploring the use of research evidence in healthenhancing physical activity policies. Health Res Policy Syst 13: 43.

7. Hämäläinen R-M, Aro AR, Juel Lau C, Rus D, Cori L, et al. (2016) Cross-sector cooperation in health-enhancing physical activity policymaking: more potential than achievements? Health Res Policy Syst 14: 33.

8. Lau CJ, Glümer C, Spitters HPEM, Sandu P, Rus D, et al. (2015) Impact of policy game on insight and attitude to inter sectoral policy processes - EU country cases. Eur J Public Health 25: s3.

9. Bertram M, Radl-Karimi C, Loncarevic N, Thøgersen M, Skovgaard T, et al. (2016) Planning Locally Tailored Interventions on Evidence Informed Policy Making - Needs Assessment, Design and Methods. Health Res Policy Syst 3: 2. 